\title{
LOVE WAVES IN FIBER-REINFORCED LAYER IMPERFECTLY BONDED TO MICROSTRUCTURAL COUPLE STRESS SUBSTRATE
}

\author{
Vishal Sharma, Vikas Sharma \\ Lovely Professional University, Department of Mathematics, Phagwara, Punjab, India \\ e-mail: lpuvishal1@gmail.com; vikas.sharma@lpu.co.in
}

\begin{abstract}
Interior of the Earth is quite complex and it shows many heterogeneities in the form of microstructures. It is difficult to model the Earth in mathematical formulation of a problem, yet it is always desirable that the proposed model should be the nearest approximation of the Earth. In this paper, Love waves are investigated, using a new geometrical configuration which consists of a finite thicker fiber-reinforced layer lying over a couple stress half-space having internal microstructures. The two media are assumed to be imperfectly bonded to each other at the interface. Dispersion and damping equations are derived for the propagation of Love waves in the considered model. The impact of various parameters like imperfectness at the interfacial surface, thickness of the layer, characteristic length parameter of the halfspace, direction of reinforcement are studied on the phase and damping velocities of Love waves.
\end{abstract}

Keywords: Love wave, couple stress theory, fiber-reinforced medium, characteristic length, imperfect interface

\section{Introduction}

It is a well known fact that interior of the Earth is heterogeneous, and the bonding of different layers in the interior of the Earth is not always ideal. These microstructures may be very complicated and could not be defined in the premise of classical theory of elasticity. The subject of seismology is developed by using linear theory of classical continuum mechanics. The mechanics associated with internal microstructures of the material cannot be omitted during the examination of any elasto-dynamical problem, so generalised microcontinuum theories involving additional length parameters, which take care of internal microstructures of the material, need to be preferred for dealing with such problems.

Love waves are horizontally polarized shear waves $(\mathrm{SH})$ which propagate in a layered structure, consisting of a layer of finite thickness deposited over a semi infinite substrate. These waves are generated during earthquakes or explosions, so study of these waves is crucial for theoretical seismology and earthquake engineering as data recorded for these waves is used for understanding of the internal structure of the Earth. These type of waves are also used in nondestructive evaluation techniques (Simonetti and Cawley, 2004; Ma et al., 2014) for finding surface flaws in a material through analysis of signature of signals which depend upon whether the wave meets the crack and corrosion or not. Many researchers have examined the problems of Love type waves propagating through various layered media, see Kończak (1989), Kuznetso (2014), Yang et al. (2017), Gupta et al. (2018) and Qu et al. (2018).

Classical continuum mechanics does not involve any length scale parameter into its formulation which can take care of internal microstructures of the material. These internal microstructures affect mechanical properties of the materials at the macroscopic level. This drawback of classical theory has led to the development of microcontinuum theories which involve additional 
length scale parameters to incorporate internal microstructures of materials. Consistent couple stress theory (Hadjesfandiari and Dargush, 2011) involves three material parameters $\lambda, \mu$ and $\eta$, out of which the first two are Lame parameters of classical continuum mechanics and the third parameter $(\eta)$, called the couple stress coefficient, depends upon a length scale parameter called the characteristic length of the material $l$, which accounts for internal microstructures of the material. This characteristic length is of the order of average cell size of the material. Many researchers have applied couple stress theory to investigate problems of wave propagation in elastic media under different conditions, see Sengupta and Ghosh (1974), Ottosen et al. (2000), Georgiadis and Velgaki (2003), Akgöz and Civalek (2013), Sharma and Kumar (2018), Goyal et al. (2018), Fan and Xu (2018). The wave propagation in an elastic medium depends upon properties of the material through which they propagate. The inner structure of the Earth involves many hard and soft rocks, so the interior of the Earth can be treated as a fiber-reinforced medium. Many artificial fiber-reinforced composites which are light in weight and high in strength are used in civil engineering structures. Researchers have solved problems of wave propagation using fiber-reinforced medium, see Belfield et al. (1983), Chattaraj and Samal (2013), Kakar and Kakar (2016), Lotfy et al. (2016), Vaishnav et al. (2017).

It is difficult to achieve perfect bonding at the interfacial surface between two media, due to specific reasons like thermal incompatibility of materials, defects during manufacturing process, damage and cracks. The imperfectness at the interfacial surface is modeled by assuming that stresses across the interface are continuous and proportional to the displacement jump across the interface. Bonding at interfacial surfaces between different layers of the Earth is not ideal, so to replicate the Earth in mathematical formulation of a problem, imperfect interfacial conditions at the boundary should be preferred. Many researchers have used imperfect boundary conditions to study the problems of wave propagation, see Lavrentyev and Rokhlin (1998), Otero et al. (2011), Singh et al. (2011), Cui et al. (2013), Sharma and Kumar (2017).

Dispersion of waves depends upon internal microstructures, heterogeneities and interfacial or boundary conditions. To model heterogeneities and internal microstructures of the Earth for the study of dispersion of Love waves, the authors have considered a new geometrical configuration which consists of a layer of finite width of a fiber-reinforced material lying over a couple stress substrate having internal microstructures. Further, it is assumed that the material of the layer and substrate are imperfectly bonded with each other.

\section{Formulation and solution of the problem}

Consider a layer of a fiber-reinforced medium of thickness $H$, placed over a couple stress half space with microstructures which are captured by an additional material parameter $l$, called characteristic length. The origin of the coordinate system $O(x, y, z)$ lies in the interfacial surface

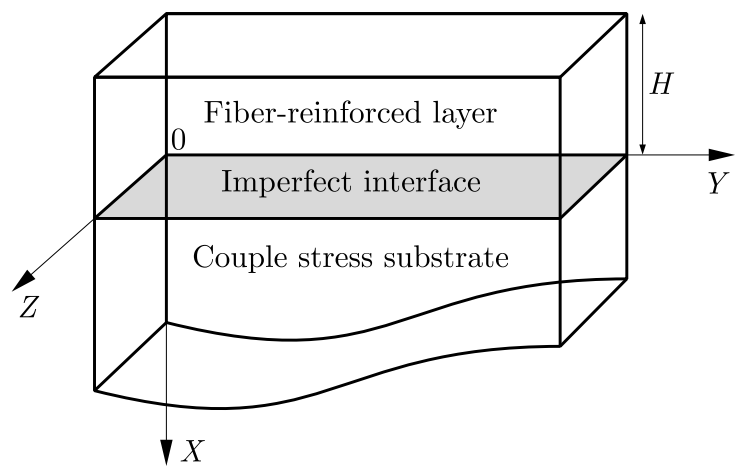

Fig. 1. Geometry of the problem 
joining the half space and the layer of the fiber-reinforced medium. We consider the $y$-axis along the direction of wave propagation, and the $x$-axis is positive vertically downwards into the half space. The interface between the layer and half space is given by $x=0$ and the free surface of the layer is $x=-H$. For Love waves, the displacement components and body forces are independent of the $z$ coordinate, so if $\left(u_{1}^{c}, u_{2}^{c}, u_{3}^{c}\right)$ are the displacement co-ordinates of a point then $u_{1}^{c}=u_{2}^{c}=0$ and $u_{3}^{c}$ is a function of the parameters $x, y$ and $t$.

\subsection{Couple stress half space}

The basic governing equation of motion and constitutive relations of consistent couple stress theory for an isotropic material in the absence of body forces (Hadjesfandiari and Dargush, 2011) are given by

$$
\left(\lambda+\mu+\eta \nabla^{2}\right) \nabla\left(\nabla \cdot u_{i}^{c}\right)+\left(\mu-\eta \nabla^{2}\right) \nabla^{2} u_{i}^{c}=\rho_{1} \frac{\partial^{2} u_{i}^{c}}{\partial t^{2}}
$$

where $\lambda$ and $\mu$ are the Lame constants, $\eta=\mu l^{2}$ is the couple-stress coefficient, $l$ is characteristic length, $\rho_{1}$ is density of the material of the elastic half space and $u_{i}^{c}$ is the displacement vector. Let us assume that $u_{i}^{c}=\left(0,0, u_{3}^{c}\right)$ and $\partial / \partial z \equiv 0$. Imposing these declared conditions, Eq. (2.1) becomes

$$
\left(\frac{\partial^{2} u_{3}^{c}}{\partial x^{2}}+\frac{\partial^{2} u_{3}^{c}}{\partial y^{2}}\right)-l^{2}\left(\frac{\partial^{4} u_{3}^{c}}{\partial x^{4}}+2 \frac{\partial^{4} u_{3}^{c}}{\partial x^{2} \partial y^{2}}+\frac{\partial^{4} u_{3}^{c}}{\partial y^{4}}\right)=\frac{1}{c_{1}^{2}} \frac{\partial^{2} u_{3}^{c}}{\partial t^{2}} \quad c_{1}^{2}=\frac{\mu}{\rho_{1}}
$$

Let $u_{3}^{c}=f(x) \exp [\mathrm{i} k(y-c t)]$ be the solution, where $k$ is the wave number and $c$ is the phase velocity. Equation (2.2) reduces to

$$
\frac{d^{4} f}{d x^{4}}-S \frac{d^{2} f}{d x^{2}}+P f=0 \quad S=2 k^{2}+\frac{1}{l^{2}} \quad P=k^{4}+\frac{k^{2}}{l^{2}}-\frac{k^{2} c^{2}}{l^{2} c_{1}^{2}}
$$

In the couple stress elastic half space, the amplitude of waves must decrease with an increase in depth, so the solution to the above differential equation becomes

$$
f(x)=A_{1} \mathrm{e}^{-b_{1} x}+A_{2} \mathrm{e}^{-b_{2} x} \quad b_{1}=\sqrt{\frac{S+\sqrt{S^{2}-4 P}}{2}} \quad b_{2}=\sqrt{\frac{S-\sqrt{S^{2}-4 P}}{2}}
$$

then

$$
u_{3}^{c}=\left(A_{1} \mathrm{e}^{-b_{1} x}+A_{2} \mathrm{e}^{-b_{2} x}\right) \mathrm{e}^{\mathrm{i} k(y-c t)}
$$

The constitutive relation in the elastic half space are given by

$$
\begin{aligned}
\sigma_{j i}^{c} & =\lambda u_{k, k}^{c} \delta_{i j}+\mu\left(u_{i, j}^{c}+u_{j, i}^{c}\right)-\eta \nabla^{2}\left(u_{i, j}^{c}-u_{j, i}^{c}\right) \\
\mu_{j i} & =4 \eta\left(\omega_{i, j}-\omega_{j, i}\right) \quad \omega_{i}=\frac{1}{2} \epsilon_{i j k} u_{k, j}^{c}
\end{aligned}
$$

Here, $\sigma_{j i}^{c}$ is the non-symmetric force stress tensor, $\mu_{j i}$ is the skew symmetric couple-stress tensor, $\delta_{i j}$ is Kronecker's delta and $\epsilon_{i j k}$ is the permutation tensor and $i, j, k=1,2,3$. From Eqs. (2.6), we get

$$
\sigma_{31}^{c}=\mu\left(\frac{\partial u_{3}^{c}}{\partial x}\right)+\eta\left(\frac{\partial^{3} u_{3}^{c}}{\partial x^{3}}+\frac{\partial^{3} u_{3}^{c}}{\partial y^{2} \partial x}\right) \quad \mu_{21}=2 \mu l^{2}\left(\frac{\partial^{2} u_{3}^{c}}{\partial x^{2}}+\frac{\partial^{2} u_{3}^{c}}{\partial y^{2}}\right)
$$

Using Eq. (2.5) in Eq. (2.7), we get

$$
\begin{aligned}
& \sigma_{31}^{c}=\left[\mu b_{1} A_{1}\left(-1-b_{1}^{2} l^{2}+k^{2} l^{2}\right) \mathrm{e}^{-b_{1} x}+\mu b_{2} A_{2}\left(-1-b_{2}^{2} l^{2}+k^{2} l^{2}\right) \mathrm{e}^{-b_{2} x}\right] \mathrm{e}^{\mathrm{i} k(y-c t)} \\
& \mu_{21}=2 \mu l^{2}\left[A_{1}\left(b_{1}^{2}-k^{2}\right) \mathrm{e}^{-b_{1} x}+A_{2}\left(b_{2}^{2}-k^{2}\right) \mathrm{e}^{-b_{2} x}\right] \mathrm{e}^{\mathrm{i} k(y-c t)}
\end{aligned}
$$




\subsection{Fiber-reinforced layer}

The constitutive equation for a fiber-reinforced transversely isotropic linear elastic medium with to a preferred direction $\mathbf{a}=\left(a_{1}, a_{2}, a_{3}\right)$ is (Belfield et al., 1983)

$$
\begin{aligned}
\tau_{i j} & =\lambda^{f} e_{k k} \delta_{i j}+2 \mu_{T} e_{i j}+\alpha\left(a_{k} a_{m} e_{k m} \delta_{i j}+e_{k k} a_{i} a_{j}\right) \\
& +2\left(\mu_{L}-\mu_{T}\right)\left(a_{i} a_{k} e_{k j}+a_{j} a_{k} e_{k i}\right)+\beta a_{k} a_{m} e_{k m} a_{i} a_{j}
\end{aligned}
$$

where $\lambda^{f}, \alpha$ and $\beta$ are elastic constants of the material, $\mu_{L}$ and $\mu_{T}$ are longitudinal and transverse shear moduli, $e_{i j}$ are components of infinitesimal strain, $\tau_{i j}$ are components of stress, $\delta_{i j}$ is Kronecker's delta and $\mathbf{a}=\left(a_{1}, a_{2}, a_{3}\right)$ is the preferred direction of the reinforcement, such that $a_{1}^{2}+a_{2}^{2}+a_{3}^{2}=1$ and $i, j, k, m=1,2,3$.

Since we assumed that propagation of a horizontally polarized surface wave is along the direction of $y$-axis, therefore, the condition in the direction of reinforcement may be taken as $\left(a_{1}, a_{2}, 0\right)$. For the propagation of Love waves, the displacement components of the fiber-reinforced medium are taken as $u_{1}^{f}=u_{2}^{f}=0, u_{3}^{f}=u_{3}^{f}(x, y, t)$ and $\partial / \partial z \equiv 0$. Here, the only non vanishing equation for the fiber-reinforced medium is

$$
\frac{\partial \tau_{31}}{\partial x}+\frac{\partial \tau_{32}}{\partial y}+\frac{\partial \tau_{33}}{\partial z}=\rho_{2} \frac{\partial^{2} u_{3}^{f}}{\partial t^{2}}
$$

where $\rho_{2}$ is density of the fiber-reinforced layer. Using the above mentioned conditions for the propagation of Love waves, Eq. (2.10) becomes

$$
\bar{p} \frac{\partial^{2} u_{3}^{f}}{\partial x^{2}}+2 \bar{q} \frac{\partial^{2} u_{3}^{f}}{\partial x \partial y}+\bar{r} \frac{\partial^{2} u_{3}^{f}}{\partial y^{2}}=\rho_{2} \frac{\partial^{2} u_{3}^{f}}{\partial t^{2}}
$$

where $\bar{p}=\mu_{T}+\left(\mu_{L}-\mu_{T}\right) a_{1}^{2}, \bar{q}=\left(\mu_{L}-\mu_{T}\right) a_{1} a_{2}$ and $\bar{r}=\mu_{T}+\left(\mu_{L}-\mu_{T}\right) a_{2}^{2}$.

Assume the solution to Eq. (2.11) as $u_{3}^{f}(x, y, t)=g(x) \exp [\mathrm{i} k(y-c t)]$. Then, we get

$$
\bar{p} \frac{d^{2} g}{d x^{2}}+2 \mathrm{i} k \bar{q} \frac{d g}{d x}+k^{2}\left(\rho_{2} c^{2}-\bar{r}\right) g(x)=0
$$

Thus, the solution to the above differential equation for $g(x)$ is given by

$$
g(x)=A_{3} \mathrm{e}^{\mathrm{i} k b_{3} x}+A_{4} \mathrm{e}^{\mathrm{i} k b_{4} x}
$$

where, $A_{3}$ and $A_{4}$ are arbitrary constants, $b_{3}=-(M+\bar{q}) / \bar{p}, b_{4}=(M-\bar{q}) / \bar{p}$ and $M=\sqrt{\bar{q}^{2}+\bar{p}\left(\rho_{2} c^{2}-\bar{r}\right)}$.

Therefore, the displacement in the fiber-reinforced medium becomes

$$
u_{3}^{f}=\left(A_{3} \mathrm{e}^{\mathrm{i} k b_{3} x}+A_{4} \mathrm{e}^{\mathrm{i} k b_{4} x}\right) \mathrm{e}^{\mathrm{i} k(y-c t)}
$$

From Eq. (2.9), we get

$$
\tau_{31}=\mathrm{i} k\left[A_{3}\left(\bar{p} b_{3}+\bar{q}\right) \mathrm{e}^{\mathrm{i} k b_{3} x}+A_{4}\left(\bar{p} b_{4}+\bar{q}\right) \mathrm{e}^{\mathrm{i} k b_{4} x}\right] \mathrm{e}^{\mathrm{i} k(y-c t)}
$$

\subsection{Boundary conditions}

Boundary conditions which are to be satisfied at the free surface of the fiber-reinforced layer and at the interfacial surface between the fiber-reinforced layer and couple stress half space are

(i) $\tau_{31}=0$ at $x=-H$,

(ii) $\tau_{31}=G\left(u_{3}^{c}-u_{3}^{f}\right)$ at $x=0$, where $G$ measures the degree of imperfectness at the interface, 
(iii) $\mu_{21}=0$ at $x=0$,

(iv) $\tau_{31}=\sigma_{31}^{c}$ at $x=0$.

Using the above mentioned boundary conditions, we get the following system of four homogeneous equations

$$
\begin{aligned}
& A_{3}\left(\bar{p} b_{3}+\bar{q}\right) \mathrm{e}^{-\mathrm{i} k b_{3} H}+A_{4}\left(\bar{p} b_{4}+\bar{q}\right) \mathrm{e}^{-\mathrm{i} k b_{4} H}=0 \\
& A_{1} G+A_{2} G-A_{3}\left[G+\mathrm{i} k\left(\bar{p} b_{3}+\bar{q}\right)\right]-A_{4}\left[G+\mathrm{i} k\left(\bar{p} b_{4}+\bar{q}\right)\right]=0 \\
& A_{1}\left(b_{1}^{2}-k^{2}\right)+A_{2}\left(b_{2}^{2}-k^{2}\right)=0 \\
& A_{1}\left[\mu b_{1}\left(1+l^{2}\left(b_{1}^{2}-k^{2}\right)\right)\right]+A_{2}\left[\mu b_{2}\left(1+l^{2}\left(b_{2}^{2}-k^{2}\right)\right)\right] \\
& \quad+A_{3}\left[\mathrm{i} k\left(\bar{p} b_{3}+\bar{q}\right)\right]+A_{4}\left[\mathrm{i} k\left(\bar{p} b_{4}+\bar{q}\right)\right]=0
\end{aligned}
$$

For non-trivial solutions of these four equations, the determinant of the coefficient matrix of four unknowns $A_{1}, A_{2}, A_{3}$ and $A_{4}$ should vanish. By solving the determinant, we get

$$
\begin{aligned}
& R\left[G\left(b_{2}^{2}-b_{1}^{2}\right)-T\right] \sin \left(k\left(b_{3}-b_{4}\right) H\right)-G T\left[\left(\bar{p} b_{3}+\bar{q}\right)-\left(\bar{p} b_{4}+\bar{q}\right) \cos \left(k\left(b_{3}-b_{4}\right) H\right)\right] \\
& \quad+\mathrm{i}\left\{R\left[G\left(b_{2}^{2}-b_{1}^{2}\right)-T\right]\left[1-\cos \left(k\left(b_{3}-b_{4}\right) H\right)\right]+G T\left(\bar{p} b_{3}+\bar{q}\right) \sin \left(k\left(b_{3}-b_{4}\right) H\right)\right\}=0
\end{aligned}
$$

where

$$
R=k\left(\bar{p} b_{3}+\bar{q}\right)\left(\bar{p} b_{4}+\bar{q}\right) \quad T=\mu\left(b_{1}-b_{2}\right)\left[b_{1} b_{2}+k^{2}-l^{2}\left(b_{1}^{2}-k^{2}\right)\left(b_{2}^{2}-k^{2}\right)\right]
$$

Separating the real and imaginary parts of Eq. (2.17), we get

$$
R\left[G\left(b_{2}^{2}-b_{1}^{2}\right)-T\right] \sin \left(k\left(b_{3}-b_{4}\right) H\right)-G T\left[\left(\bar{p} b_{3}+\bar{q}\right)-\left(\bar{p} b_{4}+\bar{q}\right) \cos \left(k\left(b_{3}-b_{4}\right) H\right)\right]=0
$$

which is the real part of Eq. (2.17) and is the dispersion equation for Love waves in the considered model, and

$$
R\left[G\left(b_{2}^{2}-b_{1}^{2}\right)-T\right]\left[1-\cos \left(k\left(b_{3}-b_{4}\right) H\right)\right]+G T\left(\bar{p} b_{4}+\bar{q}\right) \sin \left(k\left(b_{3}-b_{4}\right) H\right)=0
$$

which is the imaginary part of Eq. (2.17) and is the damping equation for Love waves in the considered model.

2.3.1. Love waves in the fiber reinforced layer over the couple stress half space with a perfectly bonded interface

Dividing Eq. (2.18) by $G$, we get

$$
R\left[\left(b_{2}^{2}-b_{1}^{2}\right)-\frac{T}{G}\right] \sin \left(k\left(b_{3}-b_{4}\right) H\right)-T\left[\left(\bar{p} b_{3}+\bar{q}\right)-\left(\bar{p} b_{4}+\bar{q}\right) \cos \left(k\left(b_{3}-b_{4}\right) H\right)\right]=0
$$

If in Eq. (2.20) we take $G \rightarrow \infty$, we get the dispersion equation for Love waves in the fiber-reinforced layer over the couple stress half space with a perfectly bonded interface as

$$
R\left(b_{2}^{2}-b_{1}^{2}\right) \sin \left(k\left(b_{3}-b_{4}\right) H\right)-T\left[\left(\bar{p} b_{3}+\bar{q}\right)-\left(\bar{p} b_{4}+\bar{q}\right) \cos \left(k\left(b_{3}-b_{4}\right) H\right)\right]=0
$$

and dividing Eq. (2.19) by $G$, we get

$$
R\left[\left(b_{2}^{2}-b_{1}^{2}\right)-\frac{T}{G}\right]\left[1-\cos \left(k\left(b_{3}-b_{4}\right) H\right)\right]+T\left(\bar{p} b_{4}+\bar{q}\right) \sin \left(k\left(b_{3}-b_{4}\right) H\right)=0
$$

where by taking $G \rightarrow \infty$ in Eq. (2.22), we get the damping equation for Love waves in the fiber-reinforced layer over the couple stress half space with the perfectly bonded interface as

$$
R\left(b_{2}^{2}-b_{1}^{2}\right)\left[1-\cos \left(k\left(b_{3}-b_{4}\right) H\right)\right]+T\left(\bar{p} b_{4}+\bar{q}\right) \sin \left(k\left(b_{3}-b_{4}\right) H\right)=0
$$




\section{Numerical results and discussion}

For the fiber reinforced layer, various material parameters are taken from Gubbins (1990) as $\mu_{L}=0.707 \cdot 10^{10} \mathrm{~N} / \mathrm{m}^{2}, \mu_{T}=0.35 \cdot 10^{10} \mathrm{~N} / \mathrm{m}^{2}$ and $\rho_{2}=1.6 \cdot 10^{3} \mathrm{~kg} / \mathrm{m}^{3}$. The material parameters for the couple stress half space, which is assumed to be made of Dionysos Marble (Vardoulakis and Georgiadis, 1997), are $\rho_{1}=2717 \mathrm{~kg} / \mathrm{m}^{3}, \mu=30.5 \cdot 10^{9} \mathrm{~N} / \mathrm{m}^{2}$ and $c_{2}=3350 \mathrm{~m} / \mathrm{s}$.

Three different values of the characteristic length parameter $l$, which are comparable with the internal cell size of granular macromorphic rock $O\left(10^{-4}\right)$ (Dionysos Marble), are taken as $l=0.0001 \mathrm{~m}, l=0.0004 \mathrm{~m}, l=0.0009 \mathrm{~m}$. All the figures have been plotted for a non-dimensional velocity $c / c_{2}$ against the dimensionless wave number $k H$, where $c_{2}=\sqrt{\mu_{T} / \rho_{2}}$.

\subsection{Effects of preferred direction of reinforcement}

To show the role of reinforced parameters on the characteristics of Love waves in the fiber-reinforced layer over a couple stress half space, five curves are plotted for the opposite nature of parameters $a_{1}$ and $a_{2}$ ( $a_{1}$ is increasing, $a_{2}$ is decreasing and $\left.a_{1}^{2}+a_{2}^{2}+a_{3}^{2}=1\right)$. Curves 1-5 have been plotted by taking $a_{1}^{2}=0, a_{2}^{2}=1 ; a_{1}^{2}=0.25, a_{2}^{2}=0.75 ; a_{1}^{2}=0.50, a_{2}^{2}=0.50$; $a_{1}^{2}=0.75, a_{2}^{2}=0.25 ; a_{1}^{2}=1, a_{2}^{2}=0$ and by keeping fixed values of other parameters such as $H=0.004 \mathrm{~m}$, characteristic length parameter $l=0.0004 \mathrm{~m}$ and the value of imperfectness parameter $G=0.35 \cdot 10^{10}$. Figure 3 is plotted using Eq. (2.18), and it shows the variation of normalized phase velocity $c / c_{2}$ against the dimensionless wave number $k H$. Figure 2 is plotted using Eq. (2.19) and keeping the same data as for Fig. 2. Figure 3 shows the variation of non-dimensional damping velocity with the dimensionless wave number. The non-dimensional phase and damping velocities of Love waves decrease sharply with an increase in the non-dimensional wave number. It can be seen that both phase and damping velocities are affected by the change in values of $a_{1}$ and $a_{2}$.

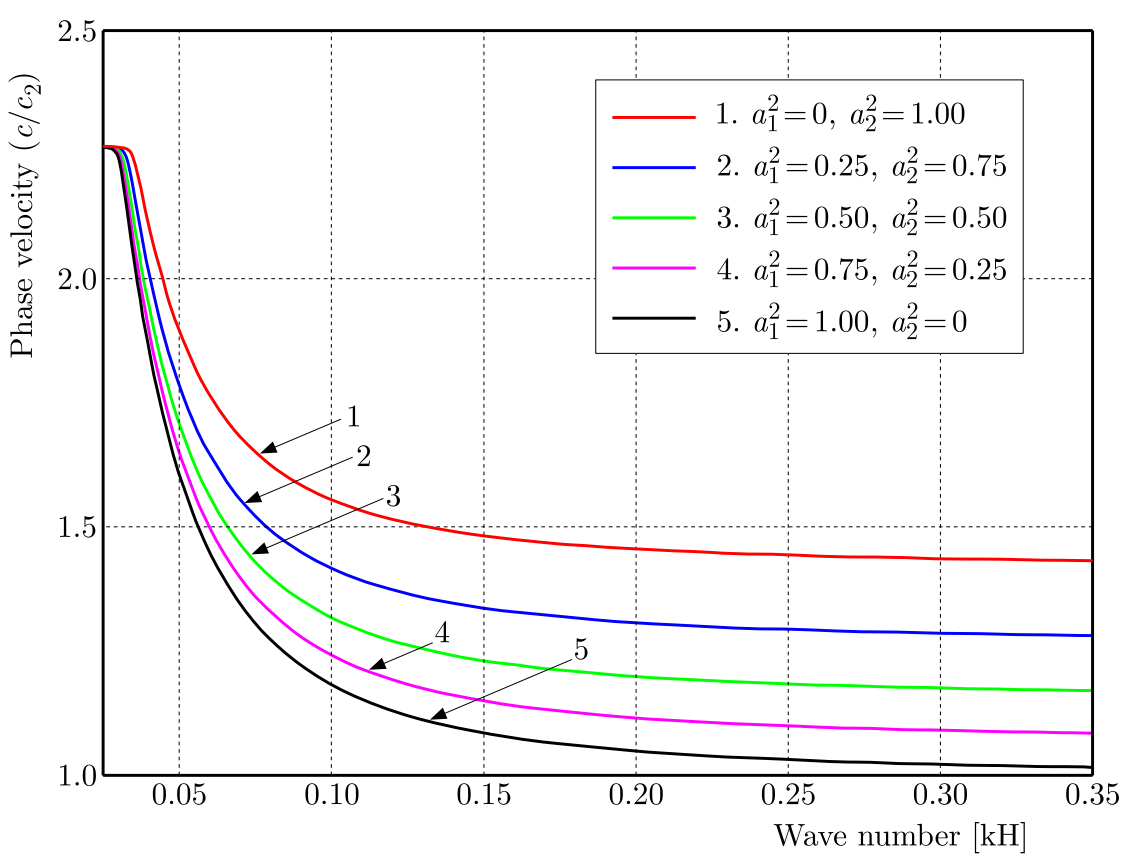

Fig. 2. Variation of normalized phase velocity $c / c_{2}$ against the dimensionless wave number $k H$ for different values of the parameters $a_{1}^{2}$ and $a_{2}^{2}$

\subsection{Effects of degree of imperfectness at the interface}

The parameter $G$, involved in the boundary conditions, defines the state of interface between the two media. For $G \rightarrow \infty$, we achieve the continuity of displacement components across the 


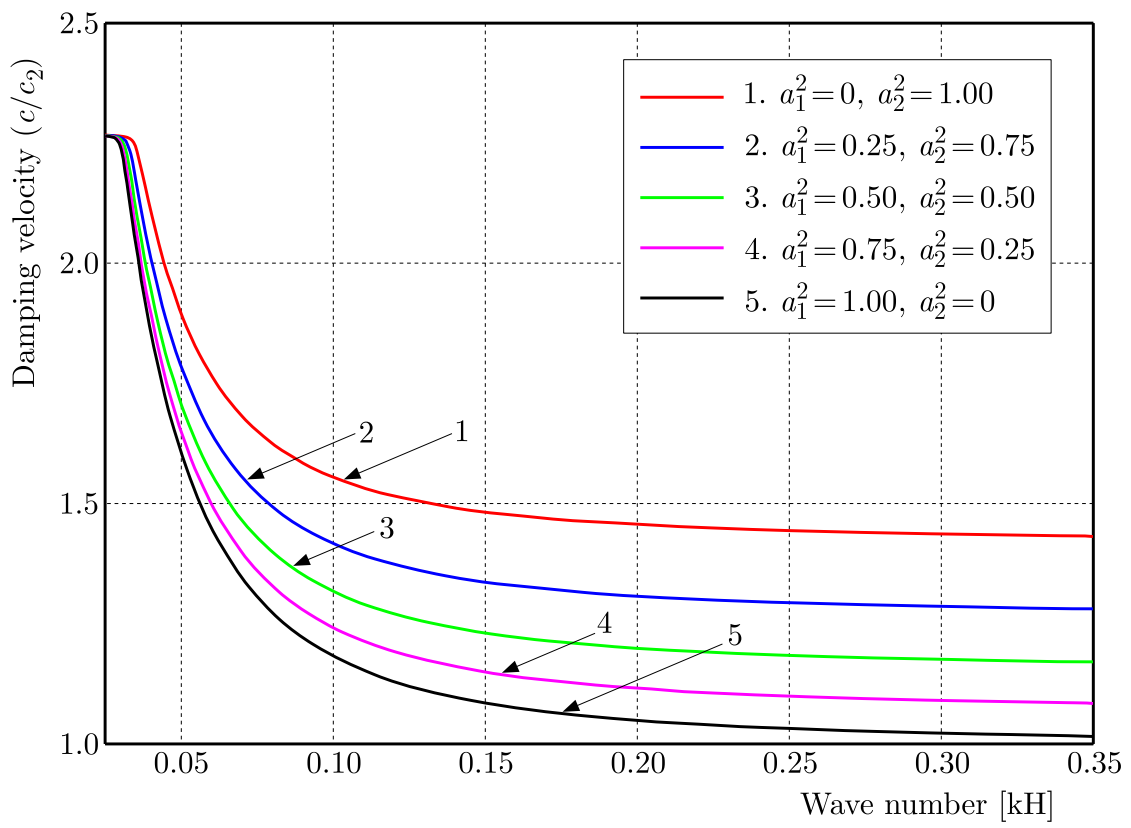

Fig. 3. Variation of normalized damping velocity $c / c_{2}$ against the dimensionless wave number $k H$ for different values of the parameters $a_{1}^{2}$ and $a_{2}^{2}$

interface, which leads to perfect bonding between the two media. Any non-zero value of the parameter $G$ leads to imperfect interface, and $G=0$ represents slippage type of interface, which means having literally no bonding among the two media. To study the role of degree of the imperfectness parameter $G$ of the interface on the propagation of Love waves in the fiberreinforced layer over the couple stress substrate, the curves are provided in Figs. 4 and 5. In these

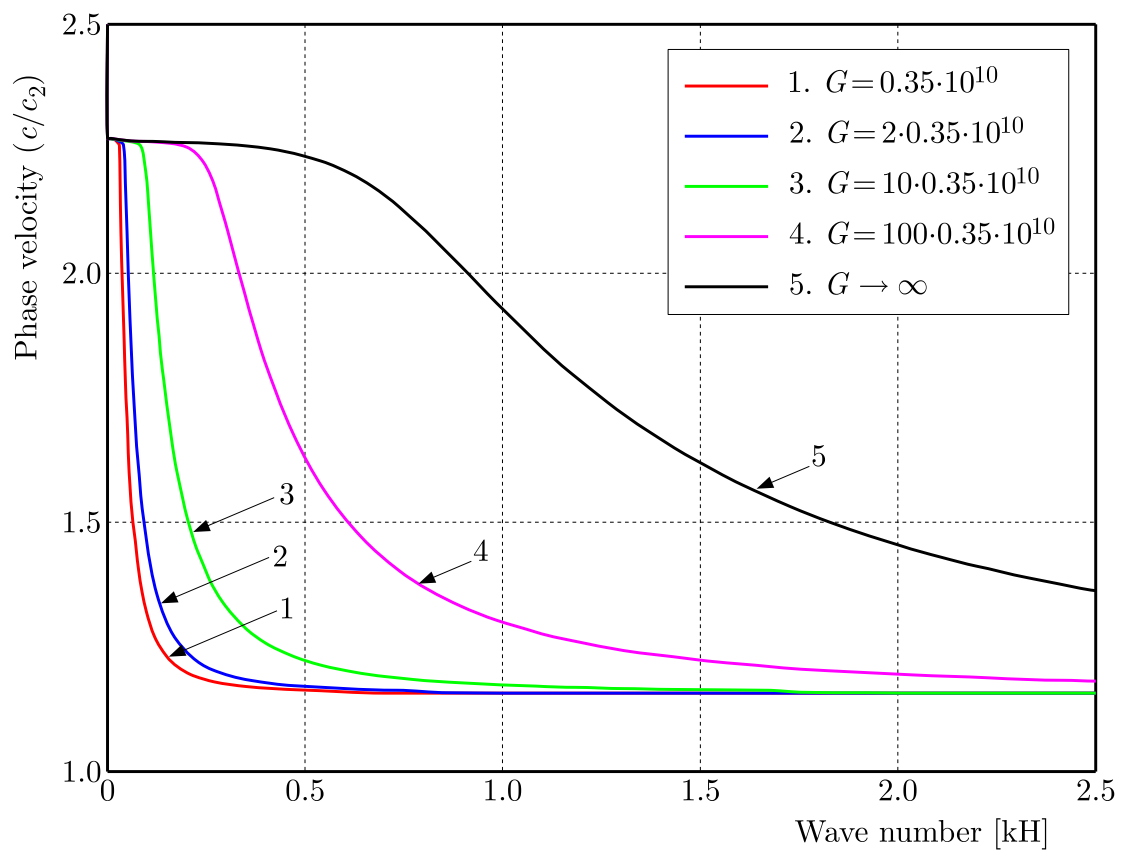

Fig. 4. Variation of normalized phase velocity $c / c_{2}$ against the dimensionless wave number $k H$ for different values of the parameter $G$

figures, we considered values of the imperfectness parameter $G$ to be $0.35 \cdot 10^{10}, 2 \times 0.35 \cdot 10^{10}$, $10 \times 0.35 \cdot 10^{10}, 100 \times 0.35 \cdot 10^{10}$ and $G \rightarrow \infty$. Here, we assumed values of the other parameters as $H=0.004 \mathrm{~m}$, characteristic length parameter $l=0.0004 \mathrm{~m}$ and $a_{1}^{2}=0.5, a_{2}^{2}=0.5$. 


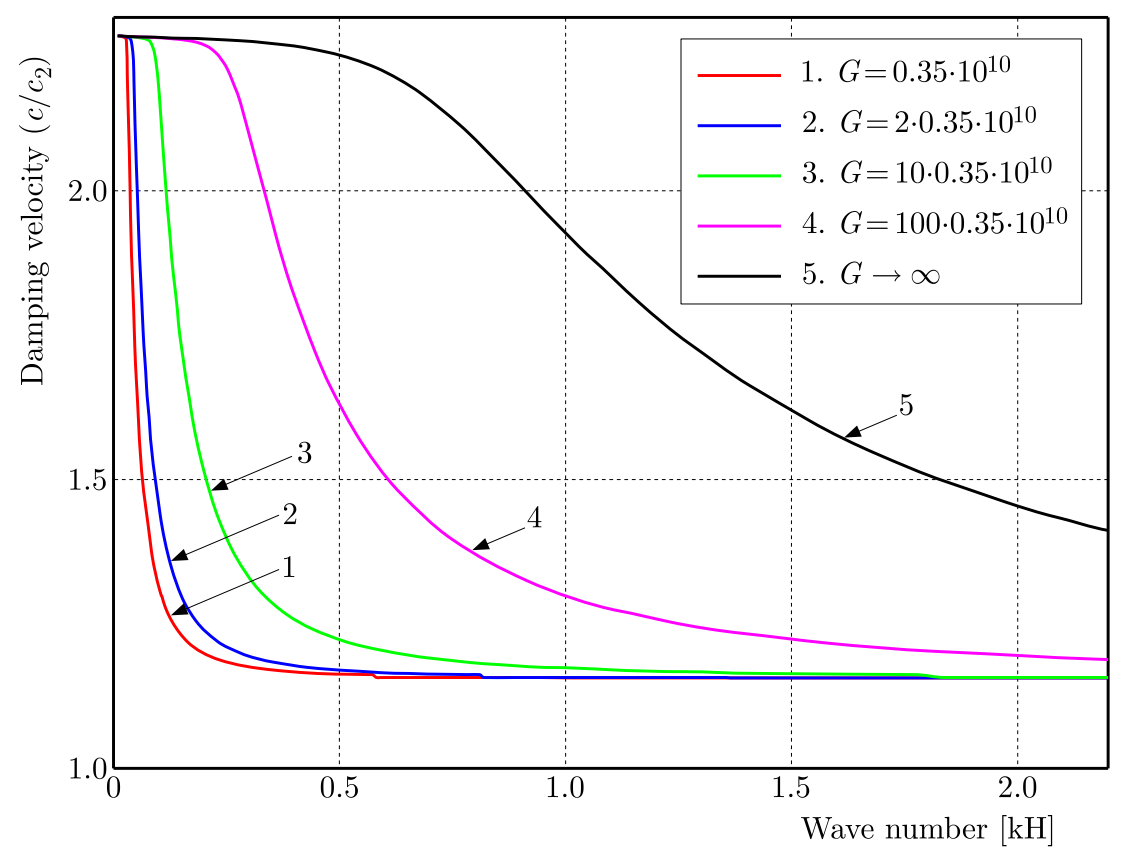

Fig. 5. Variation of normalized damping velocity $c / c_{2}$ against the dimensionless wave number $k H$ for different values of the parameter $G$

It can be observed from Figs. 4 and 5 that an increase in the parameter $G$ leads to an increase in phase and damping velocities of Love waves for any fixed value of the dimensionless wave number $k H$. Since the imperfectness is inversely proportional to $G$, so an increase in the imperfectness adversely affects the phase and damping velocities. The phase and damping velocities are maximum when the interface is perfectly bonded, that is for $G \rightarrow \infty$.

\subsection{Effects of thickness of the fiber-reinforced layer}

Figures 6 and 7 are plotted to demonstrate the impact of thickness of the layer on the phase and damping velocities of Love waves. We consider four different values of thickness of the

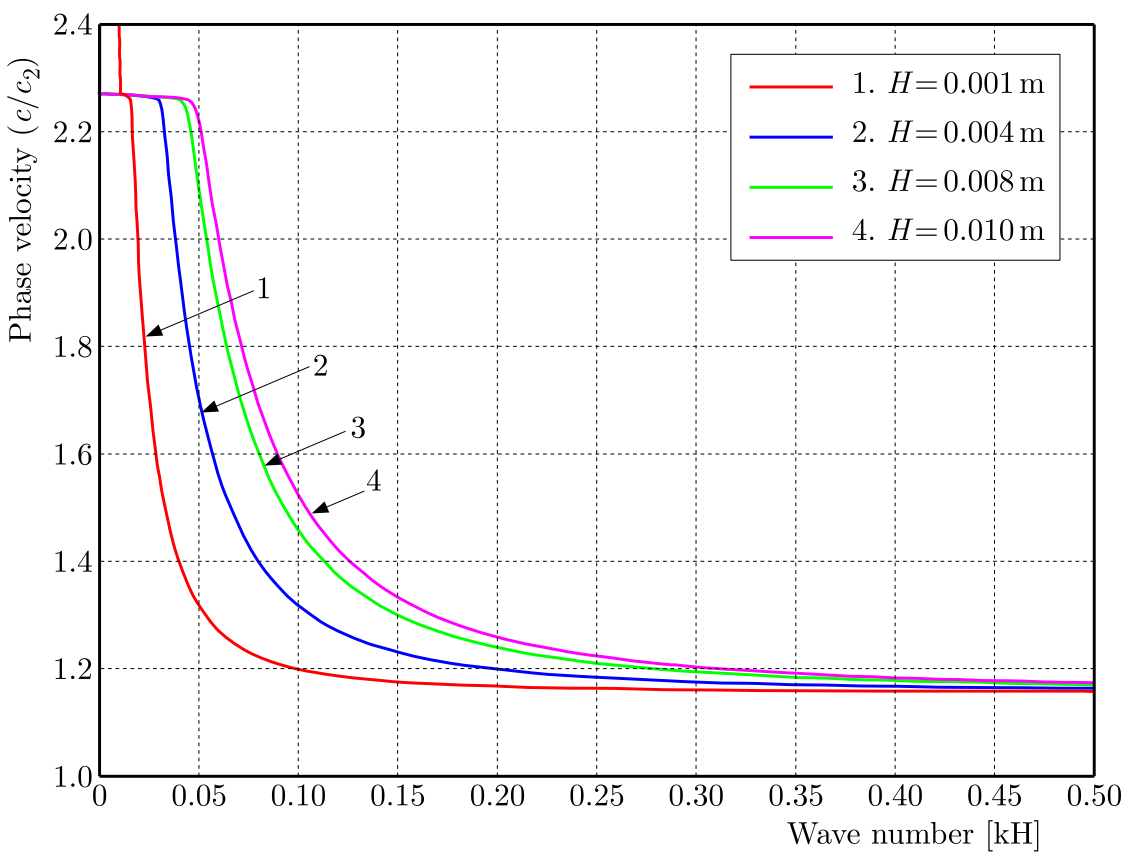

Fig. 6. Variation of normalized phase velocity $c / c_{2}$ against the dimensionless wave number $k H$ for different values of the thickness parameter $H$ 


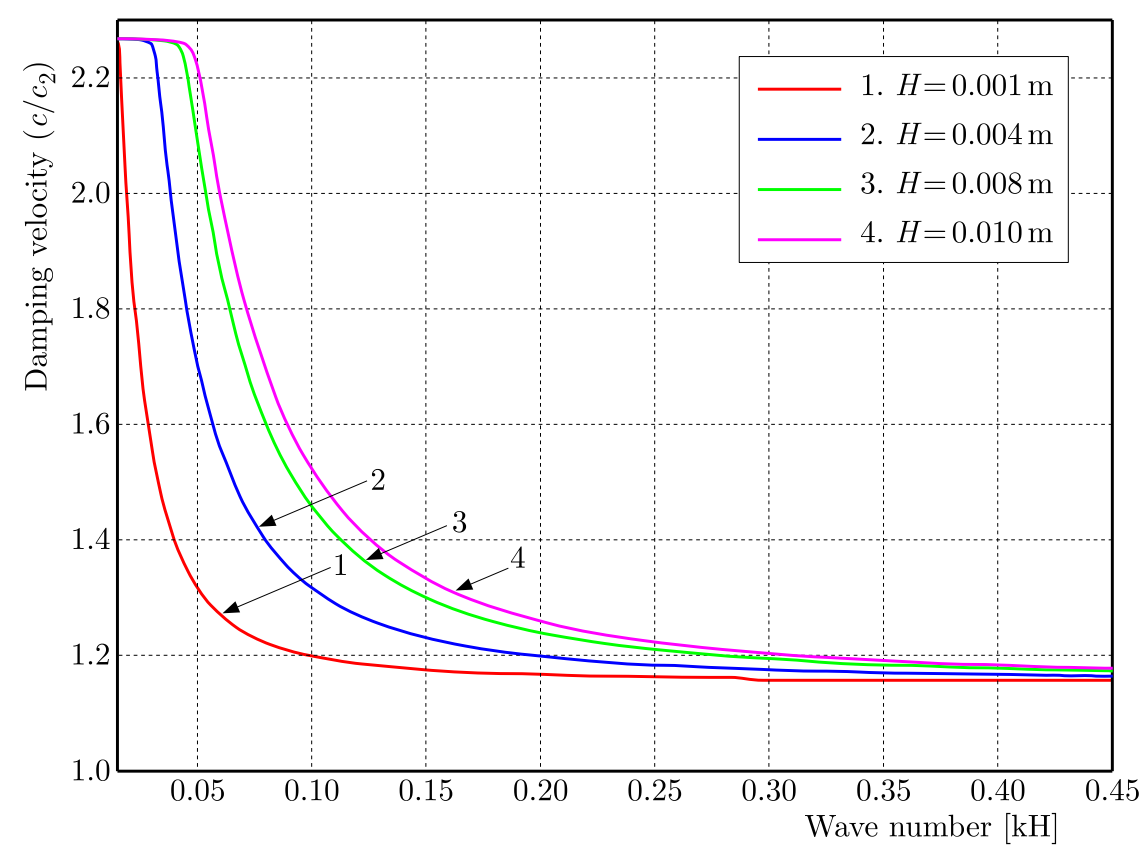

Fig. 7. Variation of normalized damping velocity $c / c_{2}$ against the dimensionless wave number $k H$ for different values of the parameter $H$

layer $H=0.001 \mathrm{~m}, 0.004 \mathrm{~m}, 0.008 \mathrm{~m}$ and $0.01 \mathrm{~m}$. The values of other parameters are taken as $l=0.0004 \mathrm{~m}, G=0.35 \cdot 10^{10}$ and $a_{1}^{2}=0.5, a_{2}^{2}=0.5$. It is observed that thickness of the layer favours phase and damping velocities. The phase and damping velocities increase with the increase in thickness of the layer at any particular wave number.

\subsection{Effects of internal microstructure of the substrate}

To observe the impact of internal microstructure of the underlying substrate on the propagation of Love waves, graphs are provided in Figs. 8 and 9. Three different values of the

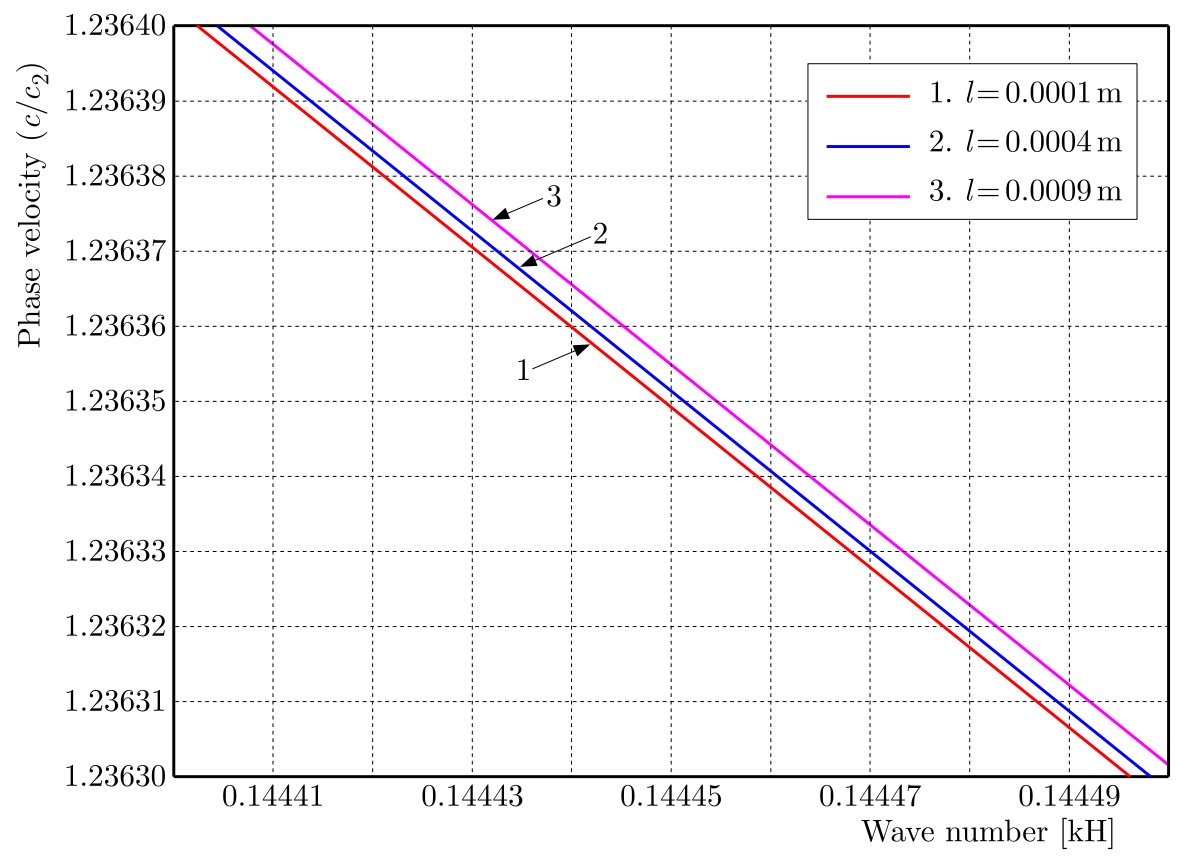

Fig. 8. Variation of normalized phase velocity $c / c_{2}$ against the dimensionless wave number $k H$ for different values of the parameter $l$ 


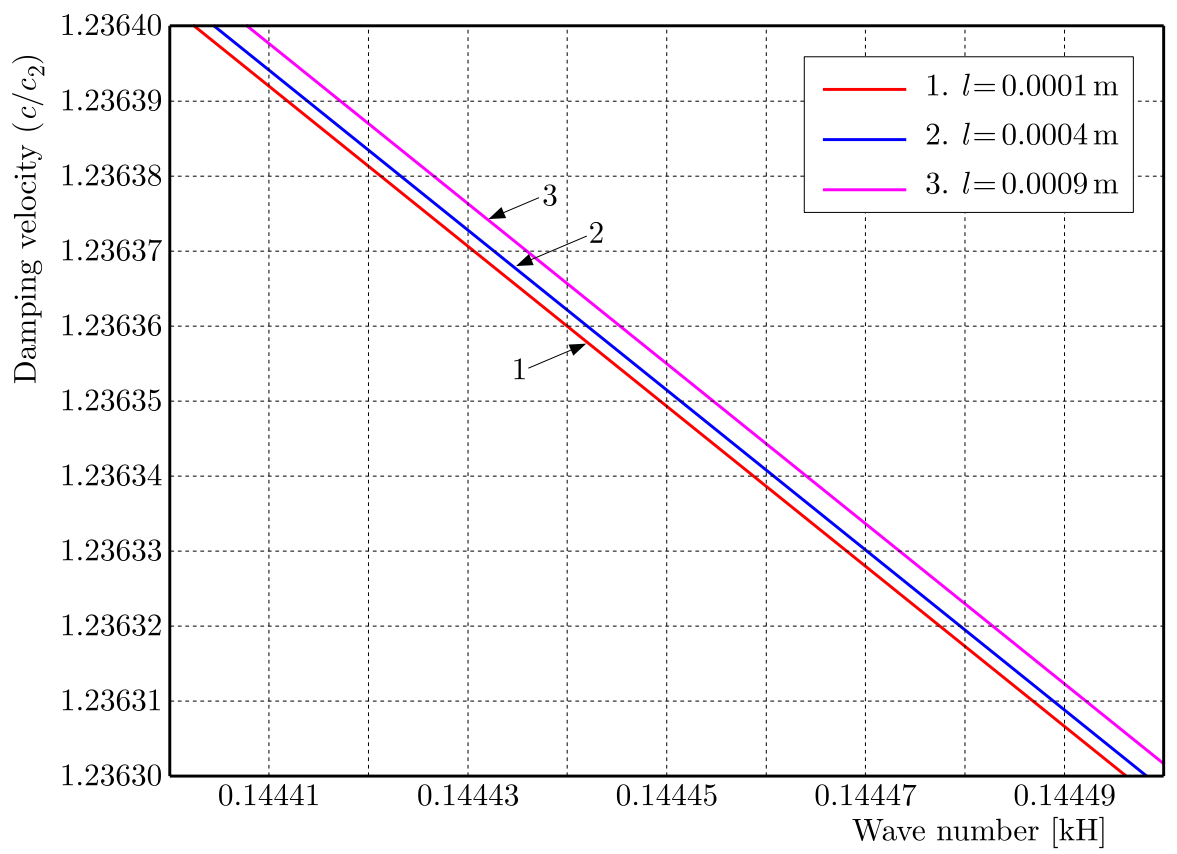

Fig. 9. Variation of normalized damping velocity $c / c_{2}$ against the dimensionless wave number $k H$ for different values of the parameter $l$

characteristic length parameter $l=0.0001 \mathrm{~m}, 0.0004 \mathrm{~m}, 0.0009 \mathrm{~m}$ are considered in these figures. The values of other parameters are taken as $H=0.004 \mathrm{~m}, G=0.35 \cdot 10^{10}$ and $a_{1}^{2}=0.5$, $a_{2}^{2}=0.5$. It can be observed that both phase and damping velocities of Love waves increase with the increase in the characteristic length parameter $l$ of the material of the substrate.

\section{Conclusion}

Love waves have numerous applications in the field of seismology, nondestructive testing techniques for material evaluation, earthquake engineering, etc. In this problem, we have studied the propagation of Love waves in a layer of finite width, which is assumed to be made of a fiber-reinforced material and is lying over a microstructural couple stress semi-infinite space. It is also assumed that both materials are imperfectly bonded to each other at the surface of contact. When both materials are perfectly bonded to each other, it is discussed as a sub-case. Dispersion and damping equations for the Love wave propagation are calculated and following major outcomes are drawn from the present analysis:

(i) Both phase and damping velocities of Love waves are decreasing with the increase in the dimensionless wave number, which shows the dependence of these velocities on the wave number. Hence, Love waves are dispersive in the considered model. It is also observed that both phase and damping velocities are affected by variations in the preferred direction of fiber-reinforcement parameters of the layer.

(ii) The parameter which corresponds to imperfectness at the interfacial surface between the two media also affects both phase and damping velocities. It is found that with the increase in imperfectness at the interfacial surface both phase and damping velocities are decreasing. Both velocities are maximum when both media are perfectly bonded to each other.

(iii) Effects of thickness of the layer on the propagation of Love waves are also studied, and it is observed that with the increase in thickness of the layer, both phase and damping velocities are increasing. Hence, thickness of the layer favors velocities of Love waves. 
(iv) The lower half space in the problem is assumed to have inner microstructures which are incorporated through a length parameter called characteristic length $l$ of the material. Effects of the characteristic length parameter are also studied, and it is found that the characteristic length parameter favors both phase and damping velocities of Love waves. Both velocities are increasing with the increase in characteristic length of the material of the lower half space.

The geometrical configuration of the problem is taken to make it consistent with the actual reality of the interior of the Earth. Since the interior of the Earth consists of different materials which can show properties of fiber-reinforced materials, the internal microstructures and layers of different materials in the interior of the Earth are not always perfectly bonded to each other. So, this problem may find some possible applications in the field of seismology or earthquake engineering. Since the lower half-space is mathematically modeled by using consistent couple stress theory, which is one of the generalized microcontinuum theories, the present analysis may provide some modifications in already established facts related to Love waves. It may further lead to modifications in the existing methods of nondestructive evaluation techniques which use Love waves for this purpose.

\section{References}

1. Akgöz B., Civalek O., 2013, Modeling and analysis of micro-sized plates resting on elastic medium using the modified couple stress theory, Meccanica, 48, 863-873, DOI: 10.1007/s11012012-9639-x

2. Belfield A.J., Rogers T.G., Spencer A.J.M., 1983, Stress in elastic plates reinforced by fibres lying in concentric circles, Journal of the Mechanics and Physics of Solids, 31, 1, 25-54

3. Chattaraj R., Samal S.K., 2013, Love waves in the fiber-reinforced layer over a gravitating porous half-space, Acta Geophysica, 61, 5, 1170-1183

4. Cui J., Du J., Wang J., 2013, Study on SH waves in piezoelectric structure with an imperfectly bonded viscoelastic layer, Joint UFFC, EFTF and PFM Symposium, 1017-1020

5. FAn H., XU L., 2018, Love wave in a classical linear elastic half-space covered by a surface layer described by the couple stress theory, Acta Mechanica, 229, 5121-5132

6. Georgiadis H.G., Velgaki E.G., 2003, High-frequency Rayleigh waves in materials with microstructure and couple-stress effects, International Journal of Solids and Structures, 40, 2501-2520

7. Goyal S., Kumar S., Sharma V., 2018, Microstructural considerations on SH-wave propagation in a piezoelectric layered structure, Journal of Theoretical and Applied Mechanics, 56, 4, 993-1004

8. Gubbins D., 1990, Seismology and Plate Tectonics, Cambridge University Press, Cambridge

9. Gupta S., Pramanik A., Ahmed M., 2018, Impact of pre-stress, inhomogeneity and porosity on the propagation of Love wave, Acta Geophysica, DOI: 10.1007/s11600-018-0185-3

10. Hadjesfandiari A.R., Dargush G.F., 2011, Couple stress theory for solids, International Journal of Solids and Structures, 48, 2496-2510

11. KAKAR R., KAKAR S., 2016, Modelling of SH-waves in a fiber-reinforced anisotropic layer over a pre-stressed heterogeneous half-space, Journal of Theoretical and Applied Mechanics, 54, 2, 463-475

12. KoŃCZAK Z., 1989, The propagation of Love waves in a fluid-saturated porous anisotropic layer, Acta Mechanica, 79, 155-168

13. KuZnetsov S.V., 2014, Dispersion of SH and Love waves, International Journal of Physics, 2, 5, $170-180$

14. Lavrentyev A.I., Rokhlin S.I., 1998, Ultrasonic spectroscopy of imperfect contact interfaces between a layer and two solids, Journal of Acoustical Society of America, 103, 2, 657-664 
15. Lotfy K., SAlem A.M., SAyed A.A., 2016, Electromagnetic field of surface waves propagation in fiber-reinforced generalized thermoelastic medium, Journal of Molecular and Engineering Materials, 3, 3-4, DOI: 10.1142/S225123731550001X

16. Ma Q., Jiao J., Hu P., Zhong X., Wu B., He C., 2014, Excitation and detection of shear horizontal waves with electromagnetic acoustic transducers for nondestructive testing of plates, Chinese Journal of Mechanical Engineering, 27, 2, 428-436

17. Otero J.A., Calás H., Rodríguez-Ramos R., Bravo-Castillero J., Aguiar A.R., MonSIVAIS G., 2011, Dispersion relations for SH waves on a magnetoelectroelastic heterostructure with imperfect interfaces, Journal of Mechanics of Materials and Structures, 6, 7-8, 969-994

18. Ottosen N.S., Ristinmaa M., Ljung C., 2000, Rayleigh waves by the indeterminate couplestress theory, European Journal of Mechanics-A/Solids, 19, 929-947

19. Qu Z., CAo X., Shen X., 2018, Properties of Love waves in functional graded saturated material, Materials, 11, 2165, DOI:10.3390/ma11112165

20. Sengupta P.R., Ghosh B., 1974, Effects of couple stresses on the propagation of waves in an elastic layer, Pure and Applied Geophysics, 112, 331-338

21. Sharma V., Kumar S., 2017, Dispersion of SH waves in a viscoelastic layer imperfectly bonded with a couple stress substrate, Journal of Theoretical and Applied Mechanics, 55, 2, 535-546

22. Sharma V., Kumar S., 2018, Dispersion of Rayleigh waves in a microstructural couple stress substrate loaded with liquid layer under the effects of gravity, Archives of Acoustics, 43, 1, 11-20

23. Simonetti F., Cawley P., 2004, On the nature of shear horizontal wave propagation in elastic plates coated with viscoelastic materials, Proceedings of the Royal Society of London-A, 460, 21972221

24. Singh J., Singh B., Ailawalia P., 2011, Propagation of waves at an imperfectly bonded interface between two monoclinic thermoelastic half-spaces, Journal of Theoretical and Applied Mechanics, 41, 3, 77-92

25. Vaishnav P.K., Kundu S., Abo-Dahab S.M., Saha A., 2017, Love wave behavior in composite fiber-reinforced structure, International Journal of Geomechanics, DOI: 10.1061/(ASCE)GM.19435622.0000945

26. Vardoulakis I., Georgiadis H.G., 1997, SH surface waves in a homogeneous gradient-elastic half-space with surface energy, Journal of Elasticity, 47, 147-165

27. Yang W., Liang X., Shen S., 2017, Love waves in layered flexoelectric structures, Philosophical Magazine, 97, 33, 3186-3209

Manuscript received December 19, 2018; accepted for print September 24, 2019 